\title{
The limits of the nuclear chart set by fission and alpha decay
}

\author{
Peter Möller \\ P. Moller Scientific Computing and Graphics, Inc., PO Box 1440, Los Alamos, NM 87544, USA
}

\begin{abstract}
I will review how our picture of heavy-element nuclear structure has evolved through remarkably simple ideas and related models. It is well known that the Bethe-Weizsäcker semi-empirical mass model had an important role in unraveling radioactive decay and element transmutation in the heavy-element region in the 1930s. A remarkable aspect is that this model could immediately after the discovery of fission be generalized to explain this phenomenon through the consideration of deformation of a charged liquid drop. Bethe and Bacher already raised the possibility that shell structure (by them calculated in terms of a single-particle oscillator potential) could give rise to noticeable deviations between results of the macroscopic mass model and experiment but limited data prevented firm conclusions. In the 1950s the single-particle models took a realistic form and also included deformation. The possibility of the existence of a relatively stable "island" of superheavy elements was raised already then. But it was not until the work by Strutinsky in the mid 1960s that a quantitative model for the nuclear potential-energy emerged in the form of the macroscopicmicroscopic model. Although new elements have been discovered at an almost steady pace since 1940 , theory indicates that we are close to the end of this era: repulsive Coulomb effects will set the limit of observable elements to near $Z=120$.
\end{abstract}

\section{Introduction}

Most insights into and understanding of nuclear properties have historically and are currently obtained in terms of surprisingly simple models. In systems which depend mainly on electron behaviour the system properties can be obtained from solving the Schrödinger equation with realistic, known Coulomb potentials. The nuclear system with its up to a few hundred nucleons leads to a many-body Schrödinger equation which cannot be solved, because the potential is based on the much more complicated nuclear forces. Therefore other types of models are used. They are often referred to in a condescending manner as phenomenological, but, actually all "models" are phenomenological. We will here focus on the remarkable insights into nuclear properties that have been obtained by the "liquid-drop model" and the single-particle model and a combination of those that still today are providing much insight about the properties of superheavy nuclei.

\footnotetext{
${ }^{a}$ e-mail: mollerinla@gmail.com
} 


\section{The liquid-drop model and limits to nuclear stability}

The liquid-drop model has its origin in the semi-empirical mass model, usually attributed to von Weizsäcker [1] and Bethe and Bacher [2]. The nuclear mass is

$$
M(Z, N, \text { shape })=M_{\mathrm{H}} Z+M_{\mathrm{n}} N-B(N, Z) .
$$

The first two terms are obviously giving the mass of all the nucleons and electrons (hydrogen mass is the sum of the a proton and an electron mass). The last term is the binding energy and accounts for the mass deficit of a nucleus relative to the sum of the masses of the separated particles. In the semi-empirical mass model it is given by

$$
B(N, Z)=+a_{\mathrm{v}} A-a_{\mathrm{s}} A^{2 / 3}-a_{\mathrm{C}} \frac{Z^{2}}{A^{1 / 3}}-a_{I} \frac{(N-Z)^{2}}{A}-\delta(A) .
$$

This simple expression gives the nuclear binding energy, which for a heavy nucleus is roughly $1600 \mathrm{MeV}$ (8 MeV per nucleon) to an accuracy of one percent. The terms represent in order volume energy, surface energy, Coulomb energy, symmetry energy and a pairing correction. The notation $Z, N$, and $A$ stand for proton number, neutron number and total nucleon number, respectively. The model was highly useful in interpreting the decay chains following element transmutations. However, in some experiments in which uranium was bombarded with neutrons a confusing number of radioactive decay products, which were difficult to reconcile with properties given by the semi-empirical mass model, were observed. This all was explained when Hahn and Strassmann [4] identified barium in the products following neutron irradiation of uranium and Meitner and Frisch [5] suggested one could think of the nucleus as a deformable charged liquid drop that had split into two smaller nuclei of about equal size in fission. It then turned out that the simple semi-empirical mass model could be generalized to describe this division and, just a few months later, Bohr and Wheeler suggested the following generalization [6]

$$
B(N, Z, \alpha)=+a_{\mathrm{v}} A-a_{\mathrm{s}} A^{2 / 3} B_{\mathrm{s}}(\alpha)-a_{\mathrm{C}} \frac{Z^{2}}{A^{1 / 3}} B_{\mathrm{C}}(\alpha)-a_{I} \frac{(N-Z)^{2}}{A}-\delta(A) .
$$

Only the Coulomb and surface energies depend on deformation. $B_{\mathrm{s}}(\alpha)$ and $B_{\mathrm{C}}(\alpha)$ are the ratios of the surface and Coulomb energies at deformation $\alpha$ to that for spherical shape. With this simple model one can show that stability with respect to fission decreases with increasing proton number and is completely lost at proton numbers somewhat above $Z=100$. Bohr and Wheeler showed this by deriving Taylor expansions for the Coulomb and surface shape dependencies. If the surface shape as function of deformation (to lowest order in $\alpha$ ) is given by

$$
r(\theta)=R_{0}\left[1+\alpha_{2} P_{2}(\cos \theta)\right]
$$

then surface and Coulomb energy to lowest order Taylor expansion are given by

$$
E_{\mathrm{s}}=E_{\mathrm{s}}^{0}\left(1+\frac{2}{5} \alpha_{2}^{2}\right) \quad \text { and } \quad E_{\mathrm{C}}=E_{\mathrm{C}}^{0}\left(1-\frac{1}{5} \alpha_{2}^{2}\right) .
$$

The energy at deformation $\alpha_{2}$ relative to spherical shape can be written as

$$
E_{\mathrm{def}}\left(\alpha_{2}\right)=E_{\mathrm{C}}\left(\alpha_{2}\right)+E_{\mathrm{s}}\left(\alpha_{2}\right)-\left(E_{\mathrm{C}}^{0}+E_{\mathrm{s}}^{0}\right) .
$$

If $E_{\text {def }}$ is negative then the spherical system has no barrier with respect to fission, i.e.

$$
E_{\mathrm{def}}\left(\alpha_{2}\right)=\frac{2}{5} \alpha_{2}^{2} E_{\mathrm{s}}^{0}-\frac{1}{5} \alpha_{2}^{2} E_{\mathrm{C}}^{0}<0
$$


Table 1. Calculated values of the fissility parameter $x$ for some sample nuclei.

\begin{tabular}{cccccccc}
\hline$Z$ & 50 & 82 & 92 & 100 & 114 & 125 & 130 \\
$A$ & 124 & 208 & 238 & 252 & 298 & 328 & 335 \\
$x$ & 0.402 & 0.645 & 0.709 & 0.792 & 0.870 & 0.950 & 1.006 \\
\hline
\end{tabular}

This condition is usually written as

$$
1<\frac{E_{\mathrm{C}}^{0}}{2 E_{\mathrm{s}}^{0}}=x .
$$

With common values of the constants $\left(E_{\mathrm{s}}^{0}=17.80 A^{2 / 3}\right.$ and $\left.E_{\mathrm{c}}^{0}=0.7103 Z^{2} / A^{1 / 3}\right)$, the fissility parameter $x$ is then obtained as

$$
x=\frac{Z^{2}}{50.13 A} .
$$

In Table 1 we calculate the fissility $x$ for a few nuclides throughout the periodic system. Clearly there are no fission barriers at $Z=130$. However, already slightly above $x=0.80$ the barrier in the liquid-drop model becomes so low that one has to expect that the spontaneousfission half-lives become sufficiently short to make such a system unobservable. It is usually taken to be around $Z=104$. Thus, already in 1939 there were credible theories that showed that that there is an end to the nuclear chart and that the end is due to fission. These insights are still pretty realistic today, but have been refined because methods have been developed to model microscopic "quantum" effects and how they can be combined with the liquid drop model to allow more accurate descriptions of nuclear masses, fission barriers, and many other nuclear properties. We will now discuss this macroscopic-microscopic model in which microscopic effects are obtained from a single-particle model.

\section{Macroscopic-microscopic model and single-particle shell effects and their benchmarks}

Already Bethe and Bacher [2] considered the possibility that microscopic effects might give rise to deviations from the semi-empirical mass model. They noted that in an oscillator potential there are large gaps in the corresponding single-particle level spectrum and investigated if there were unusually large deviations between their theory and measured masses at $Z=20, N=20$, namely ${ }^{40} \mathrm{Ca}$. They found none, but concluded that the masses were not measured sufficiently accurately to observe the effect they expected. In Fig. 1 we compare masses calculated in a modern liquid-drop model [3] to measured masses [13]. We observe that there are no particularly large deviations at these nucleon numbers. But for heavier nuclei there are large deviations at the by now well-known magic numbers. Around 1950 a modern single-particle model was developed [14-16]. It showed large gaps at the nucleon numbers where there were large deviations between the semi-empirical mass model and measured masses and it also agreed with some observed ground-state spins. But it soon became clear that for large regions of the nuclear chart spins were not well explained. This observation together with the experimental observations that nuclei in these regions had large intrinsic quadrupole moments led to the suggestion that these nuclei were not spherical but deformed [17-19]. Motivated by these developments, Nilsson in 1955 generalized the spherical single-particle model to describe levels in deformed nuclei for any prescribed spheroidal shape. A large part of the work was to devise a model specification that could be implemented on computers of the day. Nilsson solved this by introducing coordinate transformations that resulted in matrices of low dimensionality about ten or so [20]. 


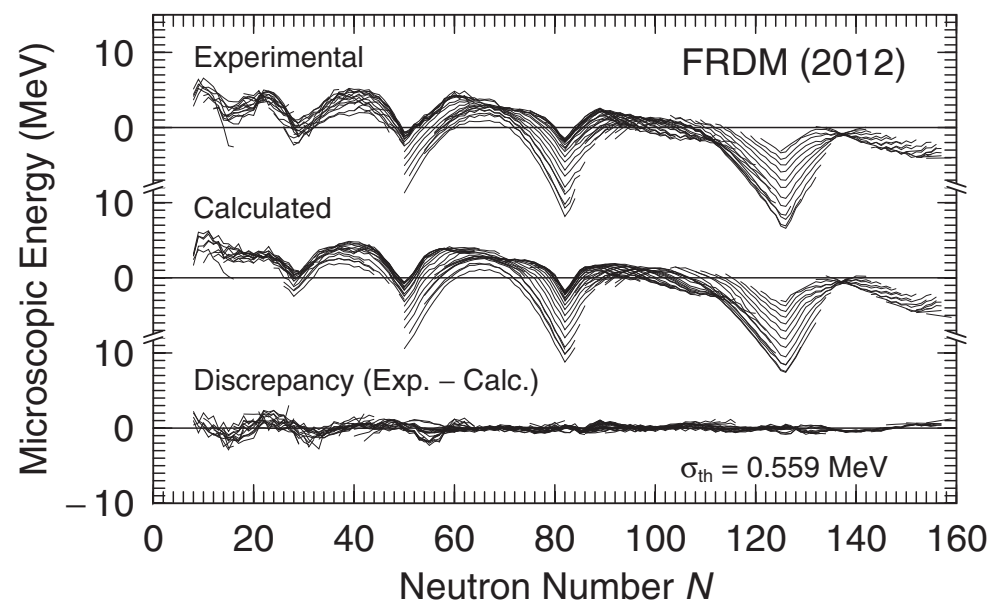

Figure 1. Top panel: difference between experimental masses and masses calculated in a modern macroscopic liquid-drop model. These are also called "experimental" shell corrections, but do depend somewhat on the theoretical macroscopic model used. Middle panel: microscopic corrections calculated by use of Strutinsky's method. Bottom panel: difference between the top and middle panel. This difference is also equivalent to the difference between experimental and calculated masses. Lines connect isotopes, full details are found in Ref. [3].

The model results for ground-state spins and low-lying level structure were shown to agree well with experimental observations [21]. To calculate the level order it was necessary to know for what deformation or nuclear shape to calculate the levels. This was done by calculating what nuclear shape has the lowest energy. The potential energy for a particular shape was calculated by summing the energy of the levels that are occupied at that shape. This was done for a succession of shapes and the ground-state shape is the shape corresponding to the minimum energy. It is the level structure here that is compared to experiment.

However, it turned out that apart from having some issues from fundamental grounds, this method of obtaining the potential energy versus shape, also did not work well in practice when the method was extended to larger deformations or additional multipole distortions. But ten years after Nilsson introduced the deformed single-particle model, Strutinsky introduced a method that combined physics from the macroscopic liquid-drop model and the singleparticle model $[22,23]$. Briefly, the potential energy for any prescribed deformation is calculated in the following manner. The energy for the charged liquid drop is calculated for this shape, somewhat simplified it is the sum of a surface and electrostatic Coulomb energy. The single-particle energies are calculated. A shell-plus-pairing correction is then obtained from the calculated single-particle spectrum. In Strutinsky's method the correction is about $-6 \mathrm{MeV}$ when there is a large gap in the spectrum such as in the neutron spectrum at $N=126$ for ${ }^{208} \mathrm{~Pb}$. Since there is also a gap in the proton levels at $Z=82$ the total shell-plus-pairing correction at spherical shape is about $-12 \mathrm{MeV}$. In situations where there is a high density of levels the shell-plus-pairing correction sum is larger than $10 \mathrm{MeV}$.

By use of the method, potential energies versus shape are calculated, the shape and energy at the minimum of this function are obtained. The energy at the minimum corresponds to the ground-state mass. In Fig. 1 we compare a modern macroscopic-microscopic mass model to experimental data, see discussion in the caption. One can gain much insight from calculated single-particles levels versus deformation, a so-called Nilsson diagram. An example is shown and discussed in Fig. 2. 


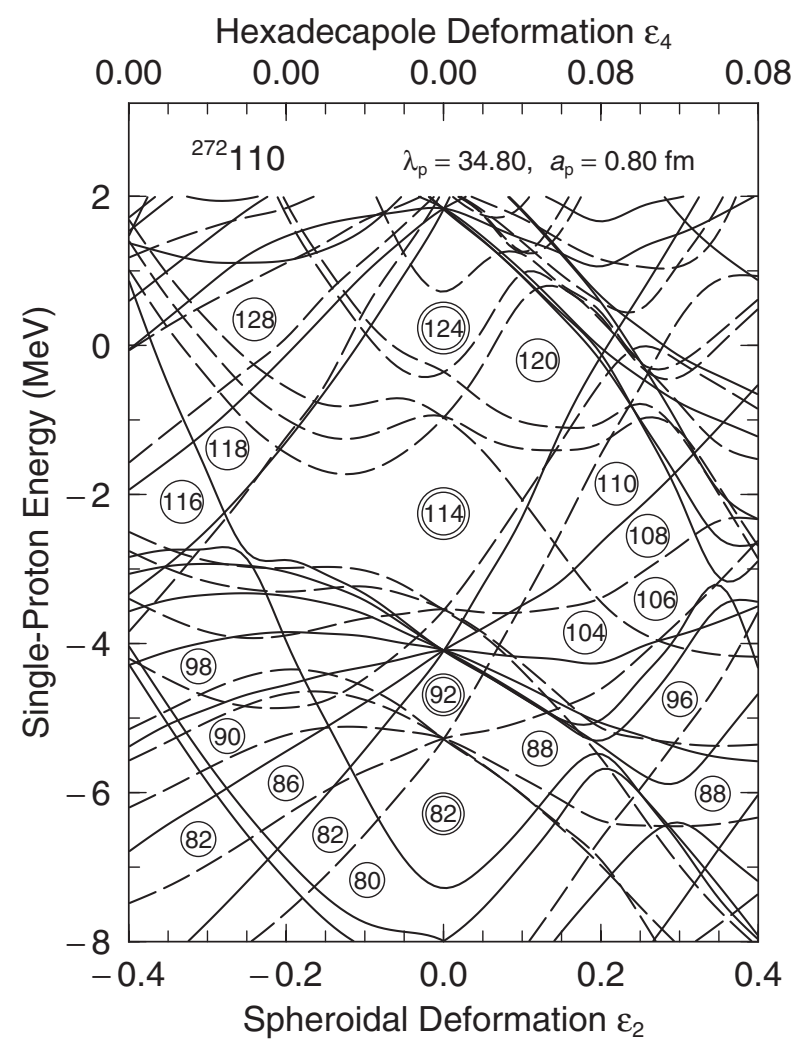

Figure 2. Calculated single-particle levels versus nuclear shape [7]. The higher multipole $\epsilon_{4}$ values are those appropriate to the nucleus ${ }^{272}$ Ds $(Z=110)$. For spherical shape we see the well-known magic proton number 82 but also a predicted gap at $Z=114$. For some time it was thought that no observable elements would exist beyond $\operatorname{Rf}(Z=104)$ and that one would need to "jump" or sail across a sea of instability to reach nuclei in the vicinity of the next predicted magic proton number 114. But by now all elements up to $Z=118$ have been observed and named. Thus, there are elements in the sea of instability. The origin of their stability are the gaps in the single-particle level diagrams for deformed shapes. The large negative shell corrections associated with these were present in early calculations [8-10] but their significance was not realized until later [11], which led to the more detailed visualization of the previous theoretical calculations [12].

No matter how well a model agrees with experimental results known at the time of publication one of several requirements to qualify as a bona fide model is that it can predict new experimental results. We have tested our FRDM (1992) mass model finalized in 1992 and published in 1995 [30] in this respect. It was adjusted to a 1989 data base of measured masses [31]. It is compared to masses in Ref. [32] that are not in the 1989 evaluation that is "new" masses in the right part of Fig. 3. The accuracy $0.642 \mathrm{MeV}$ is about the same as for the masses to which the model was adjusted and with no increase in the deviations with distance from stability. In the left part of the figure we compare to a larger data base of new masses; those that are new in the AME2003 evaluation relative to the 1989 evaluation. Now the deviation is actually substantially smaller than in the region the model was adjusted, only $0.462 \mathrm{MeV}$. An interesting feature is that many dots on the right representing large mass deviations are not present on the left, most easily noticeable on the neutron-deficient side. Since the theory did not change, this means that masses in the 1993 evaluation were either 


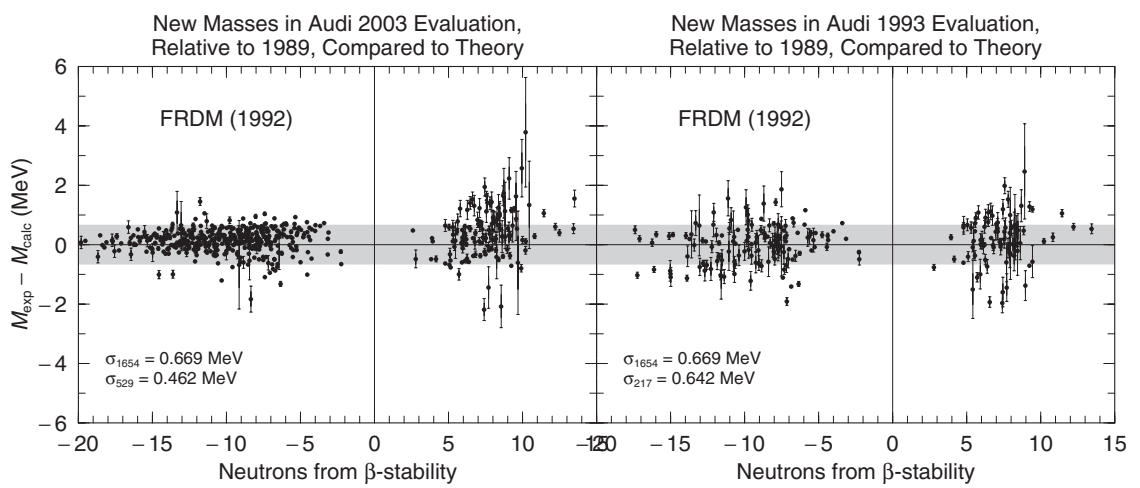

Figure 3. Comparison between masses calculated in the FRDM (1992) to measured masses that were not known when the mass table was finalized, see text for further discussion.

removed (so they are not in the 2003 evaluation) or revised by more than the error bars. One can also note that in the comparison to the right, theory and experiment did not know of each other whereas in the 2003 evaluation the evaluators knew of the FRDM(1992) since it was published in 1995 [30]. The right figure represents a double-blind result whereas the left only a single-blind result.

The calculation of fission barriers is much more complicated than the calculation of masses so we cannot review it here. Suffice to say a state-of-the-art calculation needs to calculate the potential energy for at least all major shape types that might be involved in the evolution from a single ground-state shape to separated fragments. We argue that a minimum of five shape variables are needed: elongation, left and right emerging fragment deformation (we limit our treatment to ellipsoidal shapes), neck diameter and mass division between the emerging fragments. A reasonable spacing of the shape grid points leads to calculations of potential energies for five million shapes for each nuclide considered. A slightly simplified macroscopic model, the FRLDM is used in fission calculations. Extensive details and benchmarks are given in Refs. [33-35].

\section{Superheavy nuclei}

It is unclear who first suggested the existence of a region of nuclei stabilized by magic numbers, beyond the region of increasingly unstable nuclei at the end of the nuclear chart. Gertrud Scharff-Goldhaber mentions the possibility of a stable region centered at $Z=126$ in a rather subdued fashion in Ref. [36] in 1957, so we conclude it was generally discussed already at that time. Later it was concluded that $Z=114$ was more likely [37] the next magic proton number. We give in Fig. 4 some examples of how the FRDM(1992) and FRLDM(1992) have predicted $\alpha$-decay properties of some superheavy nuclei. Recall that the heaviest nuclei to which the model was adjusted were $A \approx 260$. Note that the kinks in the experimental data are very similar in the calculated results. For $Z=113$ the origin of the kink is the gaps at $Z=108$ and $N=162$ in the deformed single-particle level spectra, see Fig. 2 for the corresponding neutron diagram from Ref. [7]. For $Z=117$ the kinks are related to changes in deformation.

It is $\alpha$-decay that usually serves to identify the observation of new elements or isotopes, but it is fission that sets the limit to what is the heaviest element that can eventually be reached. We show in Fig. 53282 calculated fission barrier heights. The calculations reproduce 

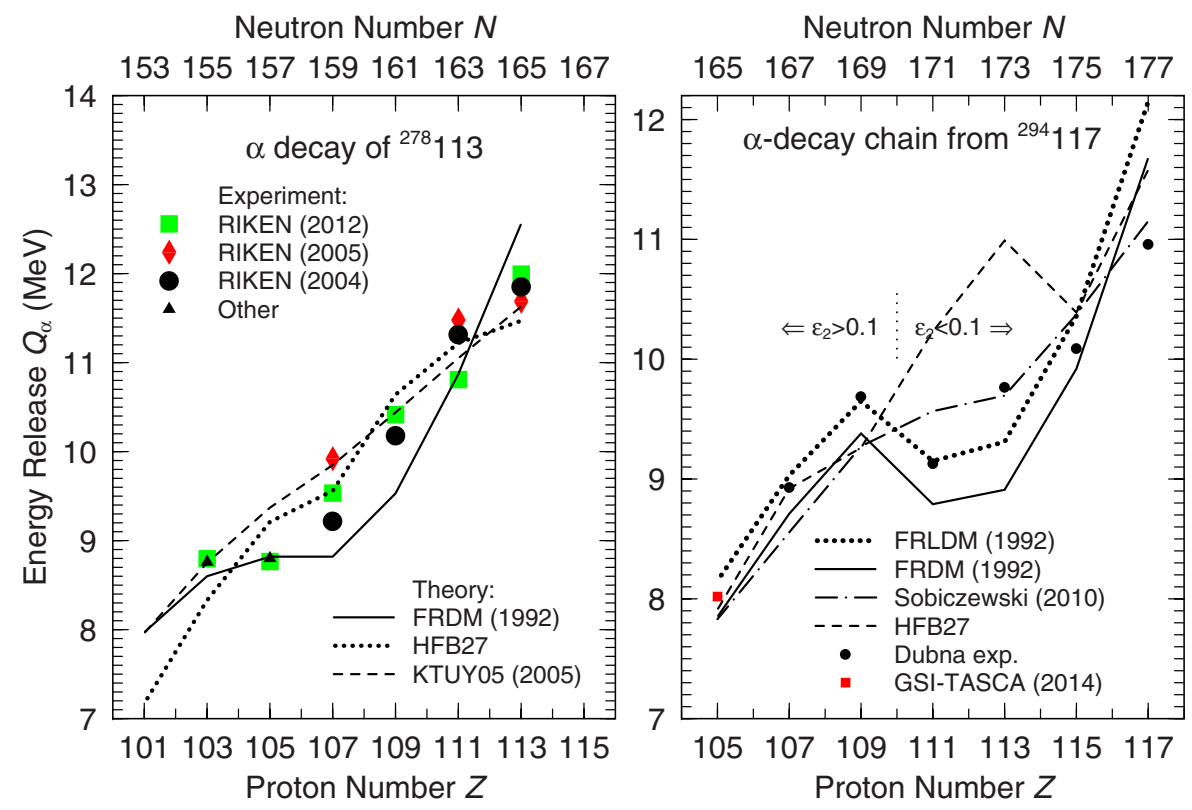

Figure 4. Measured $\alpha$-decay $Q$ values compared to calculated ground-state to ground-state $Q$ values. For $Z=113$ different colors indicate different decay chains. The measured values may not correspond to ground-state to ground-state transitions as evidenced by the differences between $Z=113$ chains. In the left figure the experimental data are from Ref. [24], HFB27 from Ref. [25] and KTUY05 from Ref. [26]. In the right figure the experimental data are from Refs. [27] and [28], Sobiczewski from Ref. [29].

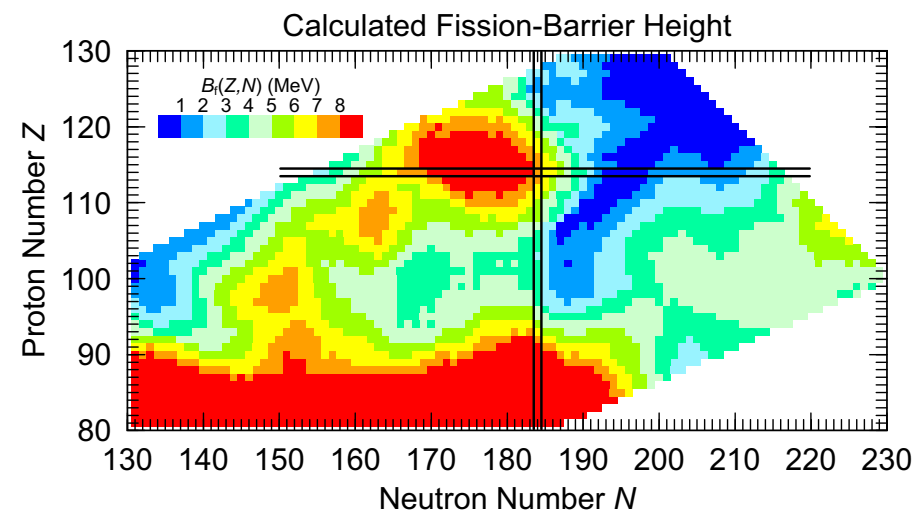

Figure 5. Calculated fission-barrier heights in the heavy region. See text and Ref. [35] for extensive discussions of barriers and the $r$-process.

the well-known regions of locally enhanced stability centered at ${ }^{252} \mathrm{Fm}(Z=100)$ and ${ }^{270} \mathrm{Hs}$ $(Z=108)$, evidenced by the calculated high barriers. For still heavier nuclei we see a larger region of high barriers, the spherical superheavy region. It is slightly offset to the north-west from the predicted doubly-magic numbers $Z=114$ and $N=184$. These calculated results and considerations suggest that it is unlikely that elements much above $Z=120$ or isotopes much beyond $N=184$ will be observed. 


\section{References}

[1] C.F. von Weizsäcker, Z. Phys. 96, 431 (1935).

[2] H.A. Bethe and R.F. Bacher, Rev. Mod. Phys. 8, 82 (1936).

[3] P. Möller, A.J. Sierk, T. Ichikawa, H. Sagawa, At. Data Nucl. Data Tables 109-110, 1 (2016).

[4] O. Hahn and F. Strassmann, Naturwiss. 27, 11 (1939).

[5] L. Meitner and O.R. Frisch, Nature 143, 239 (1939).

[6] N. Bohr and J.A. Wheeler, Phys. Rev. 56, 426 (1939).

[7] P. Möller, J.R. Nix, K.-L. Kratz, At. Data Nucl. Data Tables 66, 131 (1997).

[8] P. Möller, S.G. Nilsson, J.R. Nix, Nucl. Phys. A229, 292 (1974).

[9] P. Möller and J.R. Nix, Nucl. Phys. A361, 117 (1981).

[10] P. Möller and J.R. Nix, At. Data Nucl. Data Tables 26, 165 (1981).

[11] P. Armbruster, priv. comm., LBL Cafteria, summer 1982.

[12] R. Bengtsson, P. Möller, J.R. Nix, Jing-ye Zhang, Phys. Scr. 29, 402 (1984).

[13] M. Wang et al., Chin. Phys. C36, 1603 (2012).

[14] M. Mayer, Phys. Rev. 75, 1969 (1949).

[15] M. Mayer, Phys. Rev. 78, 16 (1950).

[16] M. Mayer, Phys. Rev. 78, 22 (1950).

[17] R. Rainwater, Phys. Rev. 79, 432 (1950).

[18] A. Bohr, Kgl. Danske Videnskab Selskab, Mat. Fys. Medd. 26 No. 14 (1952).

[19] A. Bohr and B.R. Mottelson, Kgl. Danske Videnskab Selskab, Mat. Fys. Medd. 27 No. 16 (1953).

[20] S.G. Nilsson, Kgl. Danske Videnskab Selskab, Mat. Fys. Medd. 29 No. 16 (1955).

[21] B.R. Mottelson and S.G. Nilsson, Kgl. Danske Videnskab Selskab, Mat. Fys. Skr. 1 No. 8 (1959).

[22] V.M. Strutinsky, Nucl. Phys. A95, 420 (1967).

[23] V.M. Strutinsky, Nucl. Phys. A122, 1 (1968).

[24] K. Morita et al. J. Phys. Soc. Jpn. 81, 103201 (2012).

[25] S. Goriely, N. Chamel, J.M. Pearson, Phys. Rev. C 88, 061302(R) (2013).

[26] H. Koura, T. Tachibana, M. Uno, Yamada, Prog. Theor. Phys. 113, 305 (2005).

[27] Yu.Ts. Oganessian, J. Phys. G 34, R165 (2007).

[28] J. Khuyagbaatar et al., Phys. Rev. Lett. 112, 172501 (2014).

[29] A. Sobiczewski, P. Jachimowicz, M. Kowal, Int. J. Mod. Phys. E 19, 493 (2010).

[30] P. Möller, J.R. Nix, W.D. Myers, W.J. Swiatecki, At. Data Nucl. Data Tables 59, 185 (1995).

[31] G. Audi, Midstream atomic mass evaluation, priv. comm. (1989), with four revisions.

[32] G. Audi and A.H. Wapstra, Nucl. Phys. A565, 1 (1993).

[33] P. Möller, D.G. Madland, A.J. Sierk, A. Iwamoto, Nature 409, 785 (2001).

[34] P. Möller et al., Phys. Rev. C 79, 064304 (2009).

[35] P. Möller et al., Phys. Rev. C 91, 024310 (2015).

[36] G. Scharff-Goldhaber, Nucleonics 15, 122 (1957).

[37] A. Sobiczewski, F.A. Gareev, B.N. Kalinkin, Phys. Lett. 22, 500 (1966). 